\title{
Knowledge and Practice of General Practitioners towards referral of patients to secondary care.
}

\section{S. Nimbalkar, U. Acharya, B. Vaidya, M. Shinde, K. Talati, J. Ganjiwale}

Department of Pediatrics, Pramukhswami Medical College, Karamsad, Gujarat, India. Pin -388325

Foundation for Diffusion of Innovations, Department of Interdisciplinary Research, Vadodara, India.

Central Research Services, Charutar Arogya Mandal, Karamsad, Gujarat, India. Pin - 388325

Email: somu somu@yahoo.com. Phone: 9825087842

\section{Background and Aims:}

There is wide variation in referral rates among general practitioners (GP), with studies suggesting at least fourfold variation between the highest and lowest referral rates has attracted much attention in recent years. High rates may indicate over referral, with a significant proportion of unnecessary, inappropriate referrals wasting hospital resources, while low rates might suggest under referral, resulting in increased morbidity among patients who would otherwise benefit from referral.

Much interest has been focused on the possible role of audit of referral patterns as a means of identifying inappropriate referral and so optimizing general practitioners' use of hospital resources. The GP largely controls the initial referral of patients to hospital, and so traditionally has played an important role as 'Gatekeeper'.

Referrals to secondary services form an important part of the spectrum of care available to the patients

Aim:

* To know Knowledge and Practice of General Practitioners towards referral of patients to secondary care.

- Inappropriate referrals are economically costly and may cause delays and lost opportunities for others.

\section{Methods:}

All General practitioners of Anand District would be approached for the study. After taking written informed consent, a self administered questionnaire would be given to them. Data from the proforma was be entered in Office Excel and analysis was done by STATA 14. Descriptive analysis was done to depict categorical variables.

\section{Results:}

$>90 \%$ of pediatric patients are referred from general practitioners(GPs).

$>55 \%$ GPs refer patients for respiratory conditions, $48 \%$ for gastroenteritis , $39 \%$ for seizures and $40 \%$ for other conditioned like RTI, nephritic syndrome, diabetes etc. $>58 \%$ refer due to lack of adequate resources for treatment and when they find complicated patients.

$>60 \%$ don't explain about cost of treatment.

$>76 \%$ don't explain about prognosis to relatives.

$>$ Only $45 \%$ help in contacting referral center. $44 \%$ provide referral sheet to patient.

$>75 \%$ give primary treatment before referring which includes iv fluids, antibiotics etc.

\section{Conclusion:}

Most pediatric patients are referred.

However, the GPs have poor idea of prognosis and capabilities of referral center.

There is scope for coaching the GPs for appropriate referral. 